\title{
Impairment of skeletal muscle oxygen extraction and cardiac output are matched in precapillary pulmonary hypertension
}

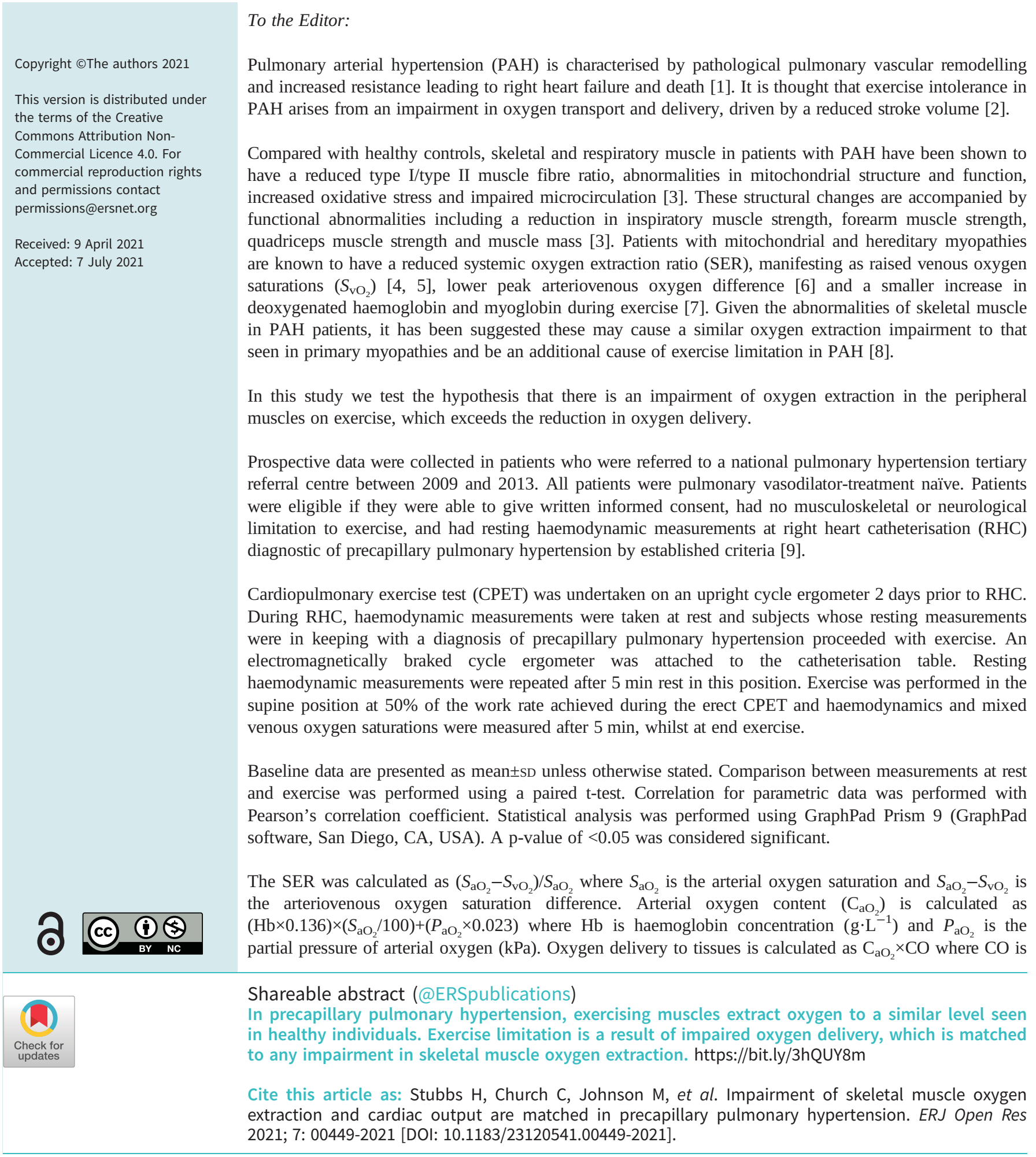


the cardiac output $\left(\mathrm{L} \cdot \mathrm{min}^{-1}\right)$. Oxygen consumption $\left(V_{\mathrm{O}_{2}}^{\prime}\right)$ is therefore calculated with the Fick equation as $\mathrm{CO} \times\left(\mathrm{C}_{\mathrm{aO}_{2}}-\mathrm{C}_{\mathrm{vO}_{2}}\right)$ where $\mathrm{C}_{\mathrm{vO}_{2}}$ is the venous oxygen content. Pulse oximetry $\left(\mathrm{S}_{\mathrm{pO}_{2}}\right)$ was used as a surrogate measure of $S_{\mathrm{aO}_{2}}$ in order to minimise the requirement for further invasive procedures.

16 patients were enrolled in the study; nine patients were diagnosed with idiopathic PAH and seven patients with chronic thromboembolic pulmonary hypertension. The mean age was 54 years. Resting and exercise measurements during RHC are presented in table 1. Mean mixed venous saturations $\left(S_{\mathrm{vO}_{2}}\right)$ fell from $62 \pm 8 \%$ at rest to $23 \pm 6.8 \%$ at peak exercise, with the lowest recorded reading of $16 \%$. The mean SER was 0.75 . A low peak exercise $S_{\mathrm{vO}_{2}}$ was strongly correlated with a lower resting $S_{\mathrm{vO}_{2}}(\mathrm{r}=0.68, \mathrm{p}<0.05)$.

This study demonstrates that in patients with precapillary pulmonary hypertension, mixed venous oxygen saturations fall markedly upon exercise. During RHC, exercise led to a fall in mean mixed venous saturations to $23 \%$ and venous partial pressure of oxygen $\left(P_{\mathrm{O}_{2}}\right)$ fell to $2.4 \mathrm{kPa}$. Whilst this study did not have a comparator group, this level of peak exercise mixed venous $P_{\mathrm{O}_{2}}$ is similar to that seen in healthy individuals (2.9 kPa) [10] and lower than that seen in patients with primary myopathy (5.5 kPa) [5]. Furthermore, the mean SER was 0.75 at peak exercise, which is in keeping with results from healthy individuals (0.72), trained individuals (0.78) (as derived from maximal exercise data) [10] and patients with heart failure with reduced ejection fraction (0.74) [8].

These results are in contrast to a retrospective review of CPETs among patients with PAH, heart failure with preserved ejection fraction and heart failure with reduced ejection fraction, which found PAH patients had a reduced SER (0.62) [8]. However, the PAH group was heterogenous in that it included patients with a normal mean pulmonary artery pressure (mPAP) at rest who developed an abnormal mPAP on exercise. Furthermore, it included PAH patients with a raised CO, affecting the linearity of the SER calculated [11].

The data shown in our study would suggest that, although there are structural and functional abnormalities of skeletal muscle in patients with precapillary $\mathrm{PH}$, any resulting impairment of oxygen extraction is matched to the reduction in oxygen delivery caused by impaired cardiac function. As a consequence, the fall seen in $S_{\mathrm{VO}_{2}}$ during exercise is similar to that seen in normal individuals. This concept is well described by WAGNER [12], with peak $V_{\mathrm{O}_{2}}^{\prime}$ limited by the concentration of inspired oxygen, the convection of oxygen to capillaries and the diffusion of oxygen across to the muscle bed. The oxygen content of blood returning from the muscles depends on the balance of these processes.

One major implication of our results is in the application of exercise training to this patient group. To maximise the benefit of peripheral muscle reconditioning, we would first need to improve oxygen delivery with appropriate disease-targeted therapy so that the balance between the two processes of oxygen delivery and extraction remains matched and optimised.

There are limitations to this study. There was a lack of a within-study comparator group. Inevitably, blood sampled from the pulmonary artery contained blood resulting from exercising and non-exercising muscles.

TABLE 1 Pulmonary haemodynamics at rest and exercise during right heart catheterisation

\begin{tabular}{|c|c|c|c|c|c|}
\hline Pulmonary artery diastolic pressure (mmHg) & $30 \pm 11$ & $42 \pm 8$ & $13 \pm 7.2$ & $<0.001$ & 1.39 \\
\hline mPAP (mmHg) & $50 \pm 12$ & $77 \pm 13$ & $27 \pm 10.4$ & $<0.001$ & 1.58 \\
\hline PAWP (mmHg) & $5.8 \pm 2$ & $9.3 \pm 3$ & $3.1 \pm 2.3$ & 0.001 & 1.5 \\
\hline Heart rate (beats $\cdot \min ^{-1}$ ) & $79 \pm 15$ & $124 \pm 17$ & $45 \pm 17.9$ & $<0.001$ & 1.63 \\
\hline Stroke volume $(\mathrm{mL})$ & $52 \pm 19$ & $56 \pm 26$ & $2.1 \pm 10.1$ & 0.45 & 1.00 \\
\hline $\mathrm{S}_{\mathrm{pO}_{2}}(\%)$ & $94 \pm 3.6$ & $89 \pm 8.2$ & $-5.0 \pm 7.1$ & 0.03 & \\
\hline $\mathrm{S}_{\mathrm{vO}_{2}}(\%)$ & $62 \pm 8$ & $23 \pm 6.8$ & $-38.3 \pm 6.5$ & $<0.001$ & \\
\hline Venous $P_{\mathrm{O}_{2}}(\mathrm{kPa})$ & $4.3 \pm 0.4$ & $2.4 \pm 0.3$ & $-1.9 \pm 0.4$ & $<0.001$ & \\
\hline
\end{tabular}


However, this would have caused an underestimate of oxygen extraction. The exercise performed during the RHC used a steady state protocol which may have not reproduced the peak exercise state at end exercise. Again, this would have led to an underestimate of oxygen extraction. Finally, a surrogate marker for $S_{\mathrm{aO}_{2}}$ was used in order to minimise participants' exposure to invasive tests, which may have led to a slight overestimation of $S_{\mathrm{aO}_{2}}$ [13].

This study shows that exercise limitation in pulmonary hypertension is primarily an impairment of oxygen delivery, as a result of a reduced CO. In precapillary pulmonary hypertension, exercising muscles extract oxygen to a similar level seen in healthy individuals, suggesting that any impairment of skeletal muscle oxygen extraction, as inferred from pathological changes in muscle biopsy specimens, is matched to an impaired oxygen delivery.

\section{Harrison Stubbs $\mathbb{0}^{1}$, Colin Church $^{1}$, Martin Johnson ${ }^{1,3}$ and Stephen Thomson ${ }^{2,3}$}

${ }^{1}$ Scottish Pulmonary Vascular Unit, Golden Jubilee National Hospital, Glasgow, UK. ${ }^{2}$ Dept of Respiratory Medicine, Queen Elizabeth University Hospital, Glasgow, UK. ${ }^{3}$ Joint last authors.

Corresponding author: Harrison Stubbs (harrison.stubbs@ggc.scot.nhs.uk)

Provenance: Submitted article, peer reviewed.

Conflict of interest: H. Stubbs has nothing to disclose. C. Church has nothing to disclose. M. Johnson reports personal fees from Actelion, PVRI and MSD, outside the submitted work. S. Thomson has nothing to disclose.

\section{References}

1 Humbert M, Guignabert C, Bonnet S, et al. Pathology and pathobiology of pulmonary hypertension: state of the art and research perspectives. Eur Respir J 2019; 53: 1801887.

2 Holverda S, Gan CT-J, Marcus JT, et al. Impaired stroke volume response to exercise in pulmonary arterial hypertension. J Am Coll Cardiol 2006; 47: 1732-1733.

3 Riou M, Pizzimenti M, Enache I, et al. Skeletal and respiratory muscle dysfunctions in pulmonary arterial hypertension. J Clin Med 2020; 9: 410.

4 Linderholm $\mathrm{H}$, MüllerT R, Ringqvist $\mathrm{T}$, et al. Hereditary abnormal muscle metabolism with hyperkinetic circulation during exercise. Acta Med Scand 1969; 185: 153-166.

5 Taivassalo $T$, Abbott $A$, Wyrick $P$, et al. Venous oxygen levels during aerobic forearm exercise: An index of impaired oxidative metabolism in mitochondrial myopathy. Ann Neurol 2002; 51: 38-44.

6 Taivassalo T, Jensen TD, Kennaway N, et al. The spectrum of exercise tolerance in mitochondrial myopathies: a study of 40 patients. Brain 2003; 126: 413-423.

7 Grassi B, Marzorati M, Lanfranconi F, et al. Impaired oxygen extraction in metabolic myopathies: detection and quantification by near-infrared spectroscopy. Muscle Nerve 2007; 35: 510-520.

8 Tolle J, Waxman A, Systrom D. Impaired systemic oxygen extraction at maximum exercise in pulmonary hypertension. Med Sci Sports Exerc 2008; 40: 3-8.

9 Simonneau G, Montani D, Celermajer DS, et al. Haemodynamic definitions and updated clinical classification of pulmonary hypertension. Eur Respir J 2019; 53: 1801913.

10 Mourtzakis M, González-Alonso J, Graham TE, et al. Hemodynamics and $\mathrm{O} 2$ uptake during maximal knee extensor exercise in untrained and trained human quadriceps muscle: effects of hyperoxia. J Appl Physiol (1985) 2004; 97: 1796-1802.

11 Wong YY, van der Laarse WJ, Vonk-Noordegraaf A. Reduced systemic oxygen extraction does not prove muscle dysfunction in PAH. Med Sci Sports Exerc 2008; 40: 1554; author reply 1555.

12 Wagner PD. Determinants of maximal oxygen transport and utilization. Annu Rev Physiol 1996; 58: 21-50.

13 Ascha M, Bhattacharyya A, Ramos JA, et al. Pulse oximetry and arterial oxygen saturation during cardiopulmonary exercise testing. Med Sci Sports Exerc 2018; 50: 1992-1997. 